\title{
A Framework for Assessing Healthcare Facilities in Low-Resource Settings: Field Studies in Benin and Uganda
}

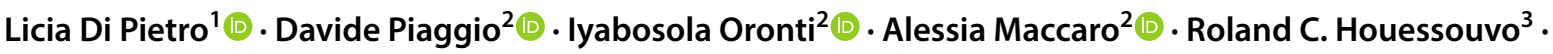

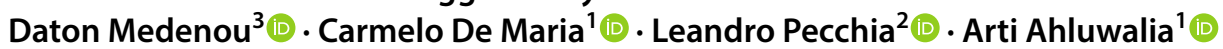

Received: 10 May 2020 / Accepted: 24 June 2020 / Published online: 8 July 2020

(c) The Author(s) 2020

\begin{abstract}
Purpose The aim of this paper is to present and validate a framework for assessing healthcare facilities in low-resource settings to collect evidence and inform policies on the harmonisation, regulation and contextualised design of medical devices. Methods A literature review and focus groups with several experts of medical device design, clinical engineering, health technology assessment and management, allowed the creation of a protocol, comprising two parts: a semi-structured interview and electrical safety measures.

Results Three hospitals were assessed in Benin and three in Uganda. All the health centres resulted to be facing typical challenges for low-resource settings, including the lack of funding, expertise, a well-established maintenance program, spare parts and consumables, and unreliable power supplies.

Conclusion As there is a paucity of information regarding low-resource settings, the proposed framework can be used by clinical or biomedical engineers to assess and thereby propose actions for improving the conditions of healthcare settings.
\end{abstract}

Keywords Clinical engineering $\cdot$ Hospital assessment $\cdot$ Healthcare facilities $\cdot$ Low-resource setting

\section{Introduction}

United Nations' (UN) 2030 Agenda for Sustainable Development includes 17 Sustainable Development Goals (SDGs), which are an urgent global call for action [1]. SDG3, Good health and well-being, strives for ensuring healthy lives globally, emphasizing the access to quality essential healthcare

Licia Di Pietro and Davide Piaggio contributed equally to this work.

Electronic supplementary material The online version of this article (https://doi.org/10.1007/s40846-020-00546-3) contains supplementary material, which is available to authorized users.

Leandro Pecchia

1.pecchia@warwick.ac.uk

1 Research Center E. Piaggio - University of Pisa, Largo Lucio Lazzarino 1, 56122 Pisa, Italy

2 School of Engineering, University of Warwick, Coventry CV4 7AL, UK

3 Department of Biomedical Engineering, Ecole Polytechnique d'Abomey-Calavi, University of Abomey-Calavi, 01 BP 2009 Cotonou, Benin services, which is currently inequitable at the expenses of low- and middle-income countries (LMICs) [1,2].

During the Sixtieth World Health Assembly, the World Health Organization (WHO) recognized the severe implications of the inappropriate provision of health technologies to support the quality of health in developing countries. The lack of appropriate technologies was recognized as a barrier to the achievement of the SDGs, specifically SDG3 with its specific target of Universal Health Coverage (UHC), which impacts on the guarantee of human rights [3].

Patients and healthcare providers in LMICs have limited access to medical devices (MDs) [4]. One barrier for the accessibility to medical technologies is the lack of funds and the high costs of imported MDs. However, if this had been the main and only problem, donations would have been a solution [5]. In this regard, WHO estimates that more than $80 \%$ of medical equipment in LMICs is donated [6], but only $10-30 \%$ of this is operating [7], due to the lack of spare parts [8], of specialized personnel [9, 10], of a good maintenance and management systems $[5,11]$ and for the harsh environmental conditions.

Other more impelling barriers derive from the fact that medical equipment is largely designed for and manufactured 
in high-income countries (HIC), following good manufacturing practices, international standards and minimum requirements, optimized for well-structured facilities, resulting difficult to be implemented in low-resource settings (LRSs) [12]. Medical equipment is designed to operate in a clean, sterile, climate-controlled environment, with a reliable main power supply, a good maintenance system, a working supply chain and expert healthcare operators. However, in LMICs, the safe and efficient use of MDs is hindered by harsh environmental and climatic conditions (e.g., high temperatures, humidity and dust), unreliable main power supplies and poor infrastructures $[13,14]$. These conditions cause frequent failures and trigger a higher demand for spare parts, which are expensive and difficult to find, making the maintenance of MDs as problematic as their acquisition [15]. As regards local hospitals LMICs, the challenges are exacerbated by the underlying structures of the buildings [16-21] (see Online material 1-3).

Given the paucity of information on the conditions of LRSs, several authors have performed reviews, interviews and observations, aimed at assessing the adequateness and appropriateness of local hospitals to deliver surgical care $[16,17]$, or intensive care [18-21] or to manage non-communicable diseases (NCDs) [22] such as hypertension [23]. In 2011, Hsia et al. [16] ran a survey based on the WHO list of essential surgical services, analysing hospitals in Kenya, Rwanda, Tanzania, Uganda and Ghana, focusing on different variables, including basic infrastructure, medicine storage capability and quality systems. The authors concluded that none of the analysed countries had the proper infrastructures for delivering surgical care. The same problems along with supply chain difficulties in terms of equipment and supplies, old infrastructures not big enough for the ever-growing population, a limited number of beds and monitored beds, were identified by Albutt et al. [17]. They also stressed the fact that the equipment, if present, is outdated and works intermittently, lacking maintenance. In some cases, the situation is exacerbated by the differences between rural and urban areas [21, 22].

Accordingly, the authors of this paper decided to implement and test a systematic framework, consisting in semistructured interviews with qualified personnel and direct measurements campaigns, for the assessment of health centres and hospitals in LRSs, given the lack of a standard protocol and of tangible, quantifiable and comparable information regarding these settings.

This paper presents the framework and its application during two field studies performed in Uganda and Benin.

\subsection{Two Countries of the Developing World: Uganda and Benin}

The UN created the Human Development Index (HDI) to evaluate the development of a country, based on different factors, including economic growth, life expectancy at birth, education and the standards of living [24]. A developing country, or a LMIC, is a country with few resources, a low HDI compared to other countries, and a gross national income per capita (GNI) below $4035 \$$.

Uganda, an Eastern African country with a GNI of $620 \$$, and Benin, a Western African country with GNI of $870 \$$, are both ranked as low-income countries (LICs) and are among the 25 poorest countries in the world [25]. Table 1 summarises some relevant data for both countries in comparison with a typical HIC.

\subsubsection{The Ugandan National Health System}

Uganda is organized into four administrative regions (i.e., Northern, Eastern, Central and Western), which are further

Table 1 Relevant data for Uganda, Benin, and Italy [26-29]

\begin{tabular}{llll}
\hline & Uganda & Benin & Italy \\
\hline Area & $241,037 \mathrm{~km}^{2}$ & $114,763 \mathrm{~km}^{2}$ & $301,338 \mathrm{~km}^{2}$ \\
HDI & 0.528 & 0.520 & 0.883 \\
Population & 42.86 million & 11.49 million & 60.48 million \\
GNI & $620 \$$ & $870 \$$ & $34,456 \$$ \\
HIV prevalence & $5.7 \%(15-49$ years old) & $1.13 \%$ (15-49 years old) & $0.3 \%$ (15-49 years old) \\
Life expectancy at birth & 60 years & 62 years & 83 years \\
Percentage of the population using an improved drinking & $79 \%$ & $77.9 \%$ (rural and urban) & $100 \%$ \\
$\quad$ water source & & & 3.95 \\
Physicians density (physicians per 1000 inhabitants) & 0.09 & 0.16 & $100 \%$ \\
Houses with centralised electricity supply & $22 \%$ & $43 \%$ & $2.06 / 7$ \\
Quality of electricity & $3.43 / 7$ & 82.38 & $5.91 / 7$ \\
Mobile phone subscriptions (per 100 people) & 57.27 & & 137 \\
\hline
\end{tabular}

${ }^{a}$ Human immunodeficiency virus 
divided into 134 districts and one city (the capital city of Kampala). The National Health System (NHS) in Uganda comprises both a private and a public sector. The public sector includes all Government of Uganda health facilities under the Ministry of Health $(\mathrm{MoH})$, and other ministries. The private health sector, which plays an important role in the delivery of health services in Uganda, includes Private Not for Profit, Private Health Practitioners, and Traditional Contemporary Medicine Practitioners. The provision of the health system is decentralised with districts and health sub-districts playing a key role in the delivery and management of health services at those levels. The health services are structured into National Referral Hospitals (NRHs) and Regional Referral Hospitals (RRHs), Fourth level General Hospital Health Centres (HCs), Third level HCs, Second level HCs, and Village Health Teams [30].

\subsubsection{The Beninese National Health System}

Benin is divided into 12 departments, 77 communes, and 546 districts, all referring to 34 sanitary zones. Its most important departments are Oumé, with the official capital (Porto-Novo), and Littoral, where the economic and administrative capital (Cotonou) is located.

Specifically, also for Benin, the NHS is decentralised, based on a pyramidal structure comprising of 3 levels:

- central: the MoH and General Secretariat are in charge of defining policies, strategies and directives;

- intermediate: the Departmental health directorates are in charge of implementing and coordinating the governmental health policies;

- peripheral, including Health zones, Commune Health Centres, village health units and private hospitals, which are the operational units.

The multifaceted realities of the departments are difficult to map out and present differences, mainly between the north and the south of the country. In addition to the ordinary health structures, there are numerous vocational hospitals spread around the country [31].

\section{Methods}

\subsection{Selection of Health Facilities}

Benin and Uganda were selected as first destinations, because of our previous experiences and the networks. Local contacts were able to link us with the hospitals, where we performed our assessment.

The selected health structures were:

- Kawolo General Hospital (Buikwe, Uganda) (H1)

- Mengo Hospital (Kampala, Uganda) (H2)

- Naguru General Hospital (Kampala, Uganda) (H3)

- Hôpital La Croix (Zinviè, Benin) (H4)

- Centre Hospitalier Universitaire (CHU) de Zone SuruLéré (Cotonou, Benin) (H5)

- CHU de Zone d'Abomey Calavi (Abomey Calavi, Benin) (H6)

\subsection{Questionnaire Preparation and Validation}

The questionnaire was drafted during focus groups among experts of medical device design, contextualised design, health technology assessment and management and clinical engineering (see Fig. 1). During these focus groups, relevant literature was used and integrated by the experts' knowledge to define the different questions and sections of the questionnaire: in particular, the sections on the general characteristics of the facility, electrical access, human resources and facility environment were based on Refs. [32, 33], those on medical electrical equipment on Refs. [34, 35] and the one on patient data management on Ref. [31].
Fig. 1 The diagram shows the methodologies used for the development and administration of the questionnaire

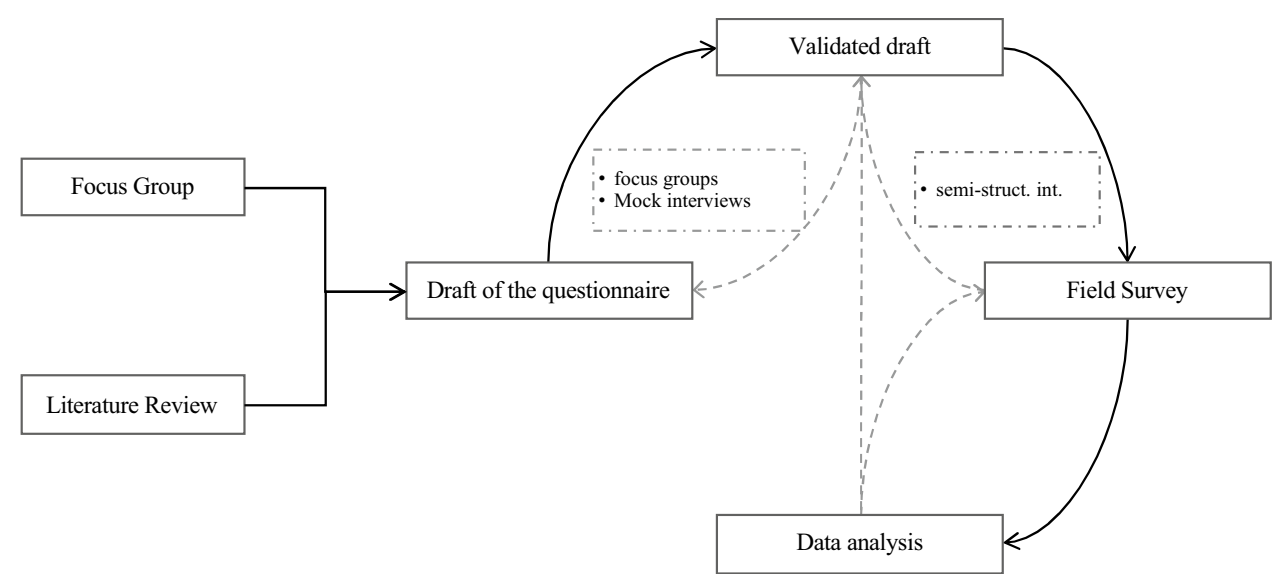


At first, the questionnaire was conceived to be online, but we realised that more relevant information could be captured if it was administered in person as a semi-structured interview (see Online material 4). The first draft of the questionnaire was circulated among the participants of the first focus group, for internal validation and possible corrections. Once the final version was ready, it was tested for further validation on an anaesthesiologist with working experience in hospitals in Sierra Leone (with Emergency).

\subsection{Electrical Safety Measures}

Electrical safety measures were carried out in accordance with IEC 62353-2015, using an electrical safety analyser (ESA620) by Fluke (see Online material 5). The MDs analysed were selected depending on availability at the health centres at the time of the assessment. Our inspection protocol included:

- Voltage and earth check on the main power supply;

- Visual inspections to check the integrity of the devices, the cables and the accessories;

- Protective earth measurement;

- Insulation resistance measurement;

- Leakage currents measurement;

- Functionality tests (e.g., ECG wave simulation).

\section{Results}

\subsection{Questionnaire}

The final questionnaire (see Online Material 6) consisted of 71 questions, organised in 9 sections: Introduction and Authorisation, Personal Information, Facility Information, Facility general characteristics, Electrical Access, Human Resources, Facility Environment, Medical Electrical
Equipment, and Database. The questions were of different type (e.g., Yes/No, multiple choice etc.) and part of them was quantitatively assessing some dimensions, others were assessing how some dimensions were perceived by the interviewee. The latter had six possible answers, based on a 5-step Likert-type scale, i.e., "Very low", "Low", "Middle", "High", "Very high", "Do not know".

The six interviewees, one per hospital, were all males (21-50 years old), had a college or university degree and were 4 biomedical engineers, 1 medical doctor and 1 nurse. All had been working in those roles for an average of 7 years.

\subsubsection{General Information About the Facility}

All the assessed hospitals are public and third-level structures (urban), but the one in Zinviè, which is private and a second-level structure (semi-urban). All the hospitals rely on piped water, but $\mathrm{H} 1$, which relies on tanker water (also available in $\mathrm{H} 2, \mathrm{H} 3$ and $\mathrm{H} 6$ ), or water from a well (also available in $\mathrm{H} 4, \mathrm{H} 6$ ). $\mathrm{H} 1$ also has structures for collecting rainwater. Only some of the structures have a functioning landline telephone $(\mathrm{H} 2, \mathrm{H} 3, \mathrm{H} 5)$. Other basic facilities are: a mobile phone $(\mathrm{H} 1, \mathrm{H} 2, \mathrm{H} 3, \mathrm{H} 5)$, a short wave radio $(\mathrm{H} 2)$, a computer (all but H4), an internet service (H2, H3, H5, H6), an ambulance (all but H5). As regards the access to electricity, all the hospitals can rely on the central supply, most of them have a generator $(\mathrm{H} 1, \mathrm{H} 2, \mathrm{H} 4, \mathrm{H} 5, \mathrm{H} 6)$ and some of them have a solar electric system $(\mathrm{H} 1, \mathrm{H} 2, \mathrm{H} 6)$ (see Table 2 for further information). All the hospitals reported a certain degree of incompatibility among the local sockets and the plugs of donated MDs (see Fig. 2).

\subsubsection{Human Resources}

All the facilities reported a chronic lack of doctors ( 1 for more than 30 patients), clinical officers ( 1 for more than 30 patients), of nurses (with an average of 1 nurse per 20

Table 2 Summary of the information and the ratings of the electrical access, reliability, and safety

\begin{tabular}{|c|c|c|c|c|c|c|}
\hline Hospital & $\begin{array}{l}\text { Power } \\
\text { outages per } \\
\text { month }\end{array}$ & $\begin{array}{l}\text { Rating of the access } \\
\text { to the main source of } \\
\text { electricity }\end{array}$ & $\begin{array}{l}\text { Rating of the quality } \\
\text { and reliability if the } \\
\text { electricity of the } \\
\text { facility }\end{array}$ & $\begin{array}{l}\text { Available and func- } \\
\text { tional systems for } \\
\text { electrical safety }\end{array}$ & $\begin{array}{l}\text { Rating of the electri- } \\
\text { cal safety in the } \\
\text { facility }\end{array}$ & $\begin{array}{l}\text { Rating of the compat- } \\
\text { ibility of the working } \\
\text { voltage and frequency } \\
\text { required for the MDs } \\
\text { and those available at } \\
\text { of the facility }\end{array}$ \\
\hline H1 & $4-6$ & Acceptable & Poor & EG & Poor & Good \\
\hline $\mathrm{H} 2$ & $1-3$ & Acceptable & Very good & EG, EN, IT & Good & Very good \\
\hline H3 & $1-3$ & Good & Acceptable & EG, EN, IT & Very good & Good \\
\hline $\mathrm{H} 4$ & $10+$ & Poor & Poor & $\mathrm{EG}, \mathrm{EN}$ & Acceptable & Poor \\
\hline H5 & $10+$ & Good & Poor & EG, EN, IT & Acceptable & Very good \\
\hline H6 & $10+$ & Acceptable & Acceptable & EG, EN & Good & Very good \\
\hline
\end{tabular}

$E G$ electrical grounding, $E N$ equipotential node, $I T$ isolation transformer 


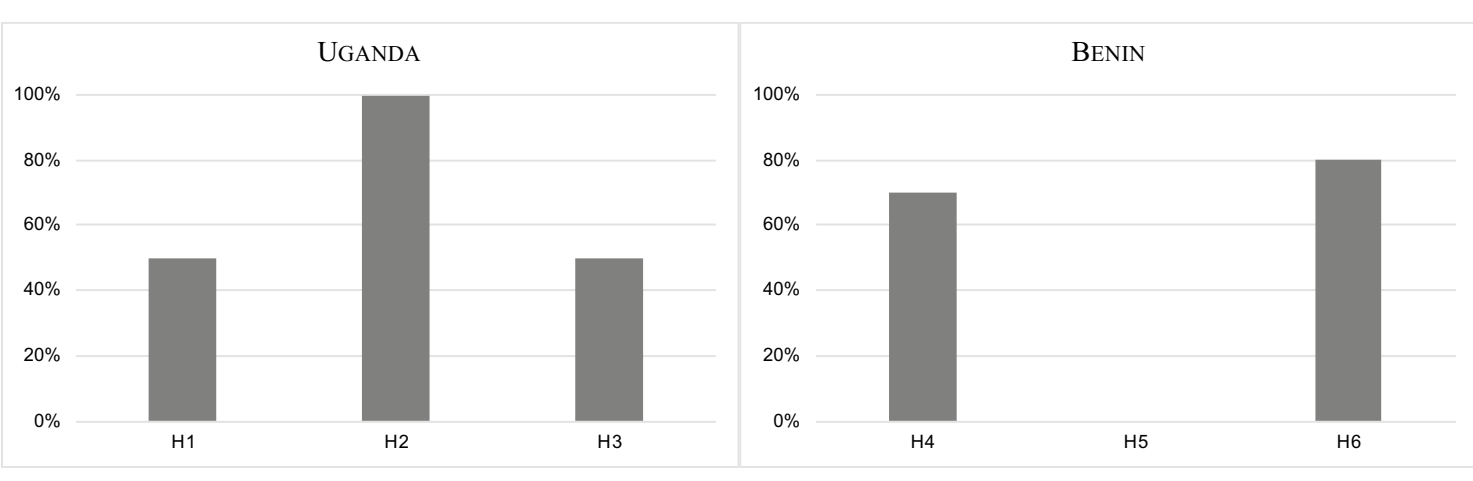

Fig. 2 The compatibility between the local sockets and the plugs of donated medical devices for Uganda and Benin

Table 3 The ratings of the insulation or distance of the structures from undue noise, dust, foul odors and smoke

\begin{tabular}{lllll}
\hline Hospital & Undue noise & Smoke & Dust & Foul odours \\
\hline H1 & Very poor & Very poor & Very poor & Very poor \\
H2 & Very poor & Very poor & Very poor & Acceptable \\
H3 & Very poor & Very poor & Very poor & Very poor \\
H4 & Acceptable & Acceptable & Poor & Acceptable \\
H5 & Good & Good & Acceptable & Good \\
H6 & Good & Poor & Acceptable & Good \\
\hline
\end{tabular}

patients), and laboratory technicians. In general, most of the interviewees judged this situation as "poor" or "very poor".

\subsubsection{Facility Environment}

In general, most of the facilities were judged by the interviewee as poorly insulated or distant from dust, smoke, and undue noise. A better situation was found regarding the insulation or distance from foul odours, the lighting, and the adequateness of ventilation (see Table 3). Similarly, the disposal of different kinds of waste (e.g., non-risk waste, infectious waste, sharps etc.) was mostly rated as "very good", apart from $\mathrm{H} 1$, for which it was rated as "very poor". All the hospitals" environments are cleaned $5+$ times per week.

\subsubsection{Medical Electrical Equipment, Maintenance, and Management}

Figure 3 shows the distribution of essential MDs and services within the six hospitals. The list is ordered from the least available piece of equipment to the most available one. Complex devices like colonoscopes, mammographs, CTscanners and X-Ray machines are a rare find, compared to blood pressure machines, thermometers, pulse-oximeters, scales, and patient monitors.

All the hospitals have a biomedical engineering department, except $\mathrm{H} 1$ and $\mathrm{H} 4$. The approach to medical device maintenance varies depending on the facility. Most of the structures that have a biomedical engineering department are in charge of the preventive and the corrective maintenance ( $\mathrm{H} 2, \mathrm{H} 5, \mathrm{H} 6)$, with the exception of $\mathrm{H} 3$ that only follows corrective maintenance practices. However, the structures that do not have a biomedical engineering department $(\mathrm{H} 1$, $\mathrm{H} 4)$ are only relying on on-call biomedical engineers/technicians, thus following only corrective maintenance practices.

The most recurrent problematics resulted to be the lack of funding, of essential medical device, of spare parts and consumables, of expertise. One of the hospitals (H1) also denounced the inexistence of a policy regulating the donation of MDs.

As regards the management, all the hospitals but one $(\mathrm{H} 4)$ have a form of inventory: 3 hospitals have a paper-based one $(\mathrm{H} 1, \mathrm{H} 2, \mathrm{H} 3)$ and 2 have or are transitioning to a computerised one (H5, H6). The most recorded information regards the year of manufacture, the type, the serial number, the year of acquisition, the class function, the date of service, the routine servicing, the reason for acquisition and the technical characteristics.

\subsection{Electric Safety Measurements}

We were able to perform these measurements in 4 hospitals ( 1 in Uganda, 3 in Benin). As regards the intensive care unit of $\mathrm{H} 2$, the inspected sockets were up to standards (voltage on the mains of $238 \mathrm{~V}$; voltage between the neutral and the ground of $0.2 \mathrm{~V}$ ). In $\mathrm{H} 2$, out of the five tested devices (i.e., 1 defibrillator, 3 patient monitors and $1 \mathrm{ECG}$ ), two of the patient monitors ${ }^{1,2}$ did not pass visual inspection because they were lacking respectively the blood pressure cuff, the ECG cables and the power cable, and the ECG cable. Moreover, the ECG cables of the defibrillator ${ }^{3}$ were not working, in fact, when inputting a simulated signal nothing was showing on the screen. All the other devices passed the inspection. Table 4 reports the findings on electrical safety.

As regards H4, the Hôpital La Croix in Benin, the sockets of the surgical room we inspected were up to standards 
Fig. 3 The distribution of essential MDs and services within the 6 hospitals. The ranges were substituted with the average value. The value 10 stands for 10 or more. Red circles individuate a low availability of the MD, yellow circles a medium availability, and green circles a high availability

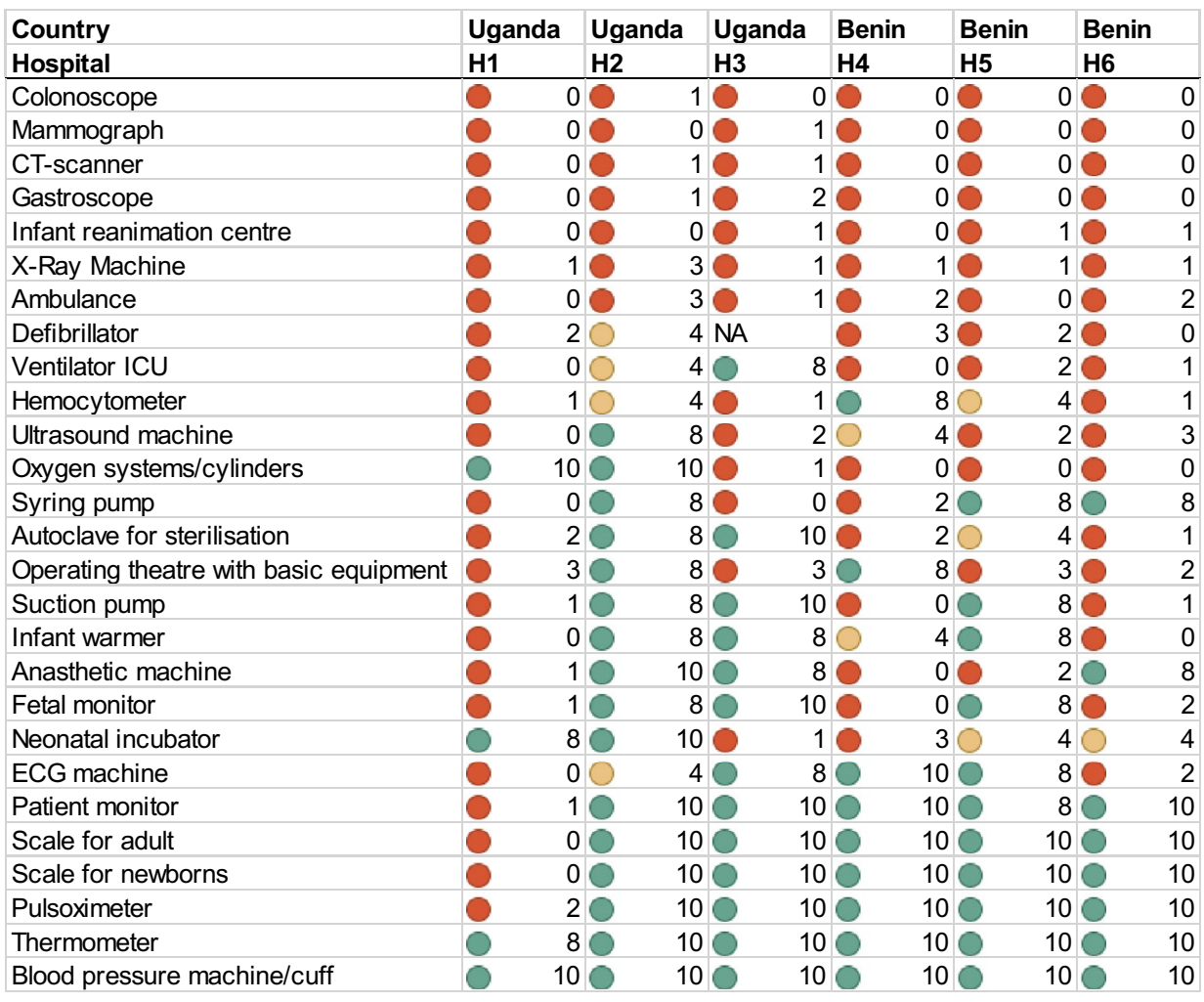

Table 4 The report of the electric safety measurements done in Mengo Hospital Intensive Care Unit (H2)

\begin{tabular}{|c|c|c|c|c|c|c|c|c|c|}
\hline \multirow[t]{2}{*}{ Hospital } & \multirow[t]{2}{*}{ Equipment } & \multirow{2}{*}{$\begin{array}{l}\text { Protective } \\
\text { earth (ohm) }\end{array}$} & \multicolumn{5}{|l|}{ Insulation } & \multicolumn{2}{|c|}{ Leakage current } \\
\hline & & & Mains-PE & AP-PE & Mains-AP & Mains-NE & AP-NE & Equipment & AP \\
\hline \multirow[t]{5}{*}{ Mengo Hospital } & Defibrillator $^{3 \mathrm{a}}$ & $\mathrm{NC}$ & Infinite & NA & NA & NA & NA & $0.2^{\mathrm{b}}$ & $5.4^{\mathrm{b}}$ \\
\hline & Patient monitor $^{2}$ & 0.075 & Infinite & NA & NA & Infinite & NA & NA & NA \\
\hline & Patient monitor ${ }^{1 \mathrm{a}}$ & - & - & - & - & - & - & - & - \\
\hline & Patient monitor & 0.065 & Infinite & Infinite & Infinite & Infinite & - & 0.3 & 5.2 \\
\hline & ECG Edan SE 1200 express & 0.043 & Infinite & 100.6 & Infinite & Infinite & 100.7 & 0.2 & 8.8 \\
\hline
\end{tabular}

$N C$ meaning normal conditions, $P E$ means protective earth, $A P$ applied part, $N E$ equipotential node

${ }^{a}$ Denotes devices that did not pass completely or partially the inspection

${ }^{b}$ Denotes measures that were taken on ECG cables as applied parts

(voltage on the mains of $240 \mathrm{~V}$; voltage between the Neutral and the Ground of $2.6 \mathrm{~V}$ ).

H6 had functional sockets in the ambulatory, however the maintenance lab and two surgical theatres had no ground, with voltages between the neutral and the ground of 89.9-134.5 V. Throughout the different buildings we noticed that the grounding system was a common problem, along with the inversed polarity of the sockets. In H5, all the inspected departments (i.e., Biochemistry, Haematology, HIV lab, new equipment room) presented troubles with the earth system (with voltages between the Neutral and the Ground between 8 and $43 \mathrm{~V}$ ), but the department of radiology. The report can be seen in Table 5 .

\section{Discussion and Conclusions}

This paper introduces a framework to assess clinical locations, specifically in LMICs, through a semi-structured interview and electrical safety measures. Such a framework, tested in Uganda and Benin, is crucial for mapping out the different realities of healthcare locations of LRSs.

Many developing countries still lack access to MDs and equipment that are appropriate for their specific clinical needs because of poor regulatory controls [36]. More importantly, what they do have is often inappropriate due to a mismatch between working conditions and design constraints. 
Table 5 The report of the electric safety measurements done in Benin (H4, H5, H6)

\begin{tabular}{|c|c|c|c|c|c|c|c|c|c|}
\hline \multirow[t]{2}{*}{ Hospital } & \multirow[t]{2}{*}{ Equipment } & \multirow{2}{*}{$\begin{array}{l}\text { Protective } \\
\text { earth (ohm) }\end{array}$} & \multicolumn{5}{|l|}{ Insulation } & \multicolumn{2}{|c|}{ Leakage current } \\
\hline & & & Mains-PE & AP-PE & Mains-AP & Mains-NE & AP-NE & Equipment & $\mathrm{AP}$ \\
\hline Hôpital la croix & Patient monitor & $1.23^{\mathrm{a}}$ & Infinite & 98.4 & Infinite & Infinite & 98.2 & 0.2 & 7.7 \\
\hline \multirow[t]{3}{*}{ Chu d'abomey Calavi } & ECG Schiller AT 102 & $0.126^{\mathrm{a}}$ & Infinite & 96.2 & Infinite & Infinite & 96.2 & 0.1 & 20.7 \\
\hline & Patient monitor $^{\mathrm{b}}$ & $1.7^{\mathrm{a}}$ & Infinite & Infinite & Infinite & Infinite & Infinite & 0.5 & 3.8 \\
\hline & Bionet fetalcare ECG & $0.34^{\mathrm{a}}$ & Infinite & Infinite & Infinite & Infinite & Infinite & 0.65 & 0.5 \\
\hline \multirow[t]{6}{*}{ Chu Suru-Lére } & Biobase Centrifuge & $0.125^{\mathrm{a}}$ & Infinite & Infinite & Infinite & Infinite & Infinite & 0.5 & NA \\
\hline & Mindray Bs200 Analyzer & $0.633^{\mathrm{a}}$ & Infinite & Infinite & Infinite & Infinite & Infinite & 34.2 & NA \\
\hline & Heamatology analyser & 0.073 & Infinite & Infinite & Infinite & Infinite & Infinite & 0.2 & NA \\
\hline & Sysmex coagulation system & $0.166^{\mathrm{a}}$ & Infinite & Infinite & Infinite & Infinite & Infinite & 0.7 & NA \\
\hline & Patient monitor & $\mathrm{NC}$ & Infinite & 99.5 & Infinite & Infinite & Infinite & 0.9 & 8 \\
\hline & Aspel ECG & $\mathrm{NC}$ & Infinite & Infinite & Infinite & Infinite & Infinite & 0.2 & 18.6 \\
\hline
\end{tabular}

$N C$ meaning normal conditions, $P E$ means protective earth, $A P$ applied part, $N E$ equipotential node

${ }^{\text {a }}$ Denotes the measures not respecting the standard

${ }^{\mathrm{b}}$ Denotes the devices for which no electrodes were available

In Africa, for example, competent authorities, who control the quality and safety of MDs, often lack adequately trained staff for consultation [37] due to limited human capacity in Biomedical Engineering [38].

We believe that the problem of existing standards is that of generalism, or of a non-inclusiveness, which does not take into consideration all the specific realities and that, for this reason, is not reachable by them. This dilemma, however, can be addressed with an inductive method, which, by examining specific situations, can inform the writing of new regulations and standards that are more inclusive and universally applicable. It is only by studying and taking into account different contexts and types of users that the design of a safe and high-quality medical device for low-resource settings can be successful [39]. It is true that many challenges are common to these settings, but it is also true that each of them has its own identity and peculiarities. According to this, open-source and collaborative methods have the potential to improve the design of needs-based MD, offering specific solutions to problems not properly considered by current standards, oriented to well-structured healthcare environments. When properly deployed, the open design paradigm has the potential to increase access to medical technologies, reducing the management, maintenance, and repair costs due to the openaccess of device blueprints [40].

In this context, UBORA, the open Biomedical Engineering e-platform for collaborative design, can be effectively used to develop safe and effective MDs, and the relative spare parts [41], the analysis of the technical needs, the risk management process, legal aspects, safety criteria and performance data, fundamental for maintaining the compliancy of the repaired devices with the medical device regulation [42].
As shown by the results of the questionnaire, all the analysed facilities are inefficiently built, with poor isolation from foul odours, smog and undue noises, and unstable and unsafe power supplies. The scarcity of essential MDs, spare parts and consumables, together with a poor maintenance system, bolstered by the chronic lack of biomedical engineers, technicians and healthcare personnel (from nurses to specialised doctors), hinders the safe and efficient care of patients, impeding universal health coverage.

The electrical safety measurements confirmed the results of the questionnaire, highlighted the common issue of too high protective earths of some MDs, and, above all, clearly proved that in Benin there are problems with the grounding systems.

One limitation of the study is that it was not always possible to test MDs, because of the lack of authorisation or because they were all being used for the care of patients.

Nevertheless, the availability of the technical staff, who allowed the measurements and the interviews on the field, made us understand the urgency for the locals of the problems highlighted in this paper. Therefore, this study is an important starting point to frame the problem and present the framework which favoured a thorough investigation in the field and facilitated the subsequent processing of the data, showing itself a suitable, exportable and repeatable tool to offer an overview of healthcare locations in specific contexts. The study will be expanded to include more hospitals around Uganda, Benin and other LMICs in order to provide the basis for promoting awareness of the issues they face and towards global policy changes for health equity.

Acknowledgements The authors would like to acknowledge all the interviewees and all the locals who helped in making this possible. Special thanks go to Joshua Kiberu, Ambrose Ziwage, Marius Yabi, 
nurse Apollinaire, Padre Hough, Yves Kinhouande, Brice Agbanglanon Adjovi A., Cosmas Obini, and Ornella Mangiacasale.

Funding DP and LP received support from the University of Warwick with two Warwick Impact Found grants supported by the EPSRC Impact Accelerator Award (EP/K503848/1 and EP/R511808/1). LDP, CDM and AA were supported the European Union's Horizon 2020 research and innovation programme under grant agreement $\mathrm{N}^{\circ} 731053$.

Availability of Data and Material Data related to this study are available upon request to the authors.

\section{Compliance with Ethical Standards}

Conflicts of interest The authors declare that they have no conflict of interest.

Ethical Approval The study was performed in accordance with ethical approval REGO-2018-2283.

Consent to Participate All the interviewees were asked and gave informed consent to participate.

Consent for Publication All the interviewees were asked and gave informed consent to publication.

Open Access This article is licensed under a Creative Commons Attribution 4.0 International License, which permits use, sharing, adaptation, distribution and reproduction in any medium or format, as long as you give appropriate credit to the original author(s) and the source, provide a link to the Creative Commons licence, and indicate if changes were made. The images or other third party material in this article are included in the article's Creative Commons licence, unless indicated otherwise in a credit line to the material. If material is not included in the article's Creative Commons licence and your intended use is not permitted by statutory regulation or exceeds the permitted use, you will need to obtain permission directly from the copyright holder. To view a copy of this licence, visit http://creativecommons.org/licenses/by/4.0/.

\section{References}

1. United Nations (UN). The sustainable development goals, March 2017. Retrieved May 5, 2020, from https://sustainabledevelopme nt.un.org/?menu $=1300$

2. Roth, L., Bempong, D., Babigumira, J. B., Banoo, S., Cooke, E., Jeffreys, D., et al. (2018). Expanding global access to essential medicines: Investment priorities for sustainably strengthening medical product regulatory systems. Globalization and Health, 14(1), 102

3. Chapman, A. R. (2016). Assessing the universal health coverage target in the Sustainable Development Goals from a human rights perspective. BMC International Health and Human Rights, 16(1), 33.

4. World Health Organization. (2010). Medical devices: Managing the mismatch: An outcome of the priority medical devices project. World Health Organization. Retrieved May 5, 2020, from https:// apps.who.int/iris/handle/10665/44407.

5. Piaggio, D., Medenou, D., Houessouvo, R. C., \& Pecchia, L. (2019). Donation of medical devices in low-income countries: Preliminary results from field studies. In International conference on medical and biological engineering (pp. 423-427). Cham: Springer.

6. Dyro, J. (Ed.). (2004). Clinical engineering handbook. Amsterdam: Elvesier.

7. World Health Organization. (2010). Barriers to innovation in the field of medical devices: Background paper 6, August 2010. World Health Organization. Retrieved May 5, 2020, from https://apps. who.int/iris/handle/10665/70457.

8. Richards-Kortum, R., \& Oden, M. (2013). Devices for lowresource health care. Science, 342(6162), 1055-1057.

9. Malkin, R. A. (2007). Design of health care technologies for the developing world. Annual Review of Biomedical Engineering, 9 , 567-587.

10. Lustick, D. R., \& Zaman, M. H. (2011). Biomedical engineering education and practice challenges and opportunities in improving health in developing countries. In 2011 Atlanta conference on science and innovation policy. IEEE.

11. Taylor, A. R., Muelenaer, P., Kochersberger, K. B., Muelenaer, A. A., Bickford, L. R., \& Redican, K. (2016). Innovating for global health: Study of healthcare technology failure in southern Malawi. Annals of Global Health, 82(3), 600-601.

12. Pecchia, L., Piaggio, D., Maccaro, A., Formisano, C., \& Iadanza, E. (2020). The inadequacy of regulatory frameworks in time of crisis and in low-resource settings: Personal protective equipment and COVID-19. Health and Technology. https://doi.org/10.1007/ s12553-020-00429-2.pdf.

13. Buzdugan, M. I., Bălan, H., \& Mureşan, D. T. (2010). An electrical power quality problem in an emergency unit from a hospitalCase study. In SPEEDAM 2010 (pp. 251-256). IEEE.

14. Rao, U., Singh, S. N., \& Thakur, C. K. (2010). Power quality issues with medical electronics equipment in hospitals. In 2010 International conference on industrial electronics, control and robotics (pp. 34-38). IEEE.

15. Malkin, R. A. (2007). Barriers for medical devices for the developing world. Expert Review of Medical Devices, 4(6), 759-763.

16. Hsia, R. Y., Mbembati, N. A., Macfarlane, S., \& Kruk, M. E. (2012). Access to emergency and surgical care in sub-Saharan Africa: The infrastructure gap. Health Policy and Planning, 27(3), 234-244.

17. Albutt, K., Yorlets, R. R., Punchak, M., Kayima, P., Namanya, D. B., Anderson, G. A., et al. (2018). You pray to your God: A qualitative analysis of challenges in the provision of safe, timely, and affordable surgical care in Uganda. PLoS ONE, 13(4), e0195986.

18. Dovlo, D., \& Karamagi, H. (2016). Life-saving hospitals-A role in UHO for Africa. Building health dreams. World Hospitals and Health Services: The Official Journal of the International Hospital Federation, 52(3), 12-16.

19. Murthy, S., Leligdowicz, A., \& Adhikari, N. K. (2015). Intensive care unit capacity in low-income countries: A systematic review. PLOS ONE, 10(1), e0116949.

20. Tumukunde, J., Sendagire, C., \& Ttendo, S. S. (2019). Development of intensive care in low-resource regions. Current Anesthesiology Reports, 9(1), 15-17.

21. Firth, P., \& Ttendo, S. (2012). Intensive care in low-income countries-A critical need. The New England Journal of Medicine, 367(21), 1974-1976.

22. Mutale, W., Bosomprah, S., Shankalala, P., Mweemba, O., Chilengi, R., Kapambwe, S., et al. (2018). Assessing capacity and readiness to manage NCDs in primary care setting: Gaps and opportunities based on adapted WHO PEN tool in Zambia. PLoS ONE, 13(8), e0200994.

23. Musinguzi, G., Bastiaens, H., Wanyenze, R. K., Mukose, A., \& Nuwaha, F. (2015). Capacity of health facilities to manage hypertension in Mukono and Buikwe districts in Uganda: Challenges and recommendations. PLOS ONE, 10(11), e0142312. 
24. United Nations Development Programme (UNDP). (2019). Human Development Report 2019. Beyond income, beyond averages, beyond today: Inequalities in human development in the $21 \mathrm{st}$ century. New York. Retrieved May 5, 2020, from http://hdr.undp. org/en/content/human-development-report-2019.

25. Harrington, J. (2018). From the Solomon Islands to Liberia: These are the 25 poorest countries in the world. USA Today. Retrieved May 5, 2020, from https://eu.usatoday.com/story/money /2018/11/29/poorest-countries-world-2018/38429473/.

26. United Nations Development Programme. (2019). Global human development indicators 2019. Retrieved May 5, 2020, from http:// hdr.undp.org/en/countries/profiles/.

27. The World Bank. World Bank open data. Retrieved May 5, 2020, from https://data.worldbank.org/.

28. The World Bank. (2017). Quality of electrical supply. Retrieved May 5, 2020, from https://govdata360.worldbank.org/indicators $/$ hf3350a8e country $=$ BRA\&indicator $=41280 \& v i z=$ line_chart \&years $=2007,2017$.

29. Central Intelligence Agency. The World Factbook. (2015). Retrieved May 5, 2020, from https://www.cia.gov/library/publi cations/the-world-factbook/fields/359.html.

30. Wikipedia Contributors. (2019). Healthcare in Uganda. In Wikipedia, the free Encyclopedia. Retrieved 17:24, May 5, 2020, from https://en.wikipedia.org/w/index.php?title=Healthcare_in_ Uganda\&oldid $=922014431$.

31. Medenou, D., Fagbemi, L. A., Houessouvo, R. C., Jossou, T. R., Ahouandjinou, M. H., Piaggio, D., et al. (2019). Medical devices in Sub-Saharan Africa: Optimal assistance via a computerized maintenance management system (CMMS) in Benin. Health and Technology, 9(3), 219-232.

32. Singh, S. (2008) Location \& Layout of Hospital. Master of Hospital Administration. [Lecture slides]. In press.

33. AIA Academy of Architecture for Health, \& Facilities Guidelines Institute. (2006). Guidelines for design and construction of hospital and health care facilities. New York: Aia Press.
34. World Health Organization. Priority medical devices. Retrieved May 5, 2020, from https://www.who.int/medical_devices/priority/ en/.

35. World Health Organization. (2011). Medical equipment maintenance programme overview: WHO Medical device technical series. World Health Organization. Retrieved May 5, 2020, from https://www.who.int/medical_devices/publications/medical-equip ment-maintenance/en/.

36. De Maria, C., Di Pietro, L., Lantada, A. D., Madete, J., Makobore, P. N., Mridha, M., et al. (2018). Safe innovation: On medical device legislation in Europe and Africa. Health Policy and Technology, 7(2), 156-165.

37. Buckley, G. J., \& Riviere, J. E. (Eds.). (2012). Ensuring safe foods and medical products through stronger regulatory systems abroad. Washington, DC: National Academies Press.

38. Lustick, D. R., \& Zaman, M. H. (2011). Biomedical engineering education and practice challenges and opportunities in improving health in developing countries. In 2011 Atlanta conference on science and innovation policy (pp. 1-5). IEEE.

39. De Maria, C., Mazzei, D., \& Ahluwalia, A. (2015). Improving African health care through open source Biomedical Engineering. International Journal on Advances in Life Sciences, 7(1), 10-19.

40. De Maria, C., Di Pietro, L., Lantada, A. D., Ravizza, A., Mridha, M., Torop, J., et al. (2019). The UBORA E-Infrastructure for Open Source Innovation in Medical Technology. In Mediterranean conference on medical and biological engineering and computing (pp. 878-882). Cham: Springer.

41. Di Pietro, L., De Maria, C., Ravizza, A., \& Ahluwalia, A. (2019). Co-design open-source medical devices: How to minimize the human error using UBORA e-infrastructure. In 201941 st annual international conference of the IEEE engineering in medicine and biology society (EMBC) (pp. 3730-3733). IEEE.

42. UBORA: Euro-Africa Open Biomedical Engineering Innovation e-platform for Innovation through Education. Retrieved May 5, 2020, from https://platform.ubora-biomedical.org/. 